\title{
含酰亚胺骨架的大环化合物合成、结构及其对阴离子的识别研究
}

\author{
王芳芳 ${ }^{a}$ 欧 敏 ${ }^{a}$ 邓雅欣 ${ }^{a}$ 由 旭 ${ }^{a}$ 张奇龙 ${ }^{b}$ 朱必学*, $a$ \\ ( ${ }^{a}$ 贵州大学大环及超分子化学重点实验室 贵阳 550025) \\ ${ }^{b}$ 贵阳医学院化学教研室 贵阳 550004)
}

\begin{abstract}
摘要 在磷酸催化作用下, 采用前体二胺 $N, N^{\prime}$-(2-胺基苯基)-2,6-二甲酰亚胺吡啶(1)和前体二醛 1,4-二(2'-甲酰苯氧基) 丁烷(2)进行缩合作用得到 $[1+1]$ Schiff 碱大环化合物 3, 进一步将 Schiff碱大环 3 还原得到饱和大环 4. 并采用 ${ }^{1} \mathrm{H} N \mathrm{NR}$, IR，质谱和元素分析等技术对大环 3 和 4 的组成进行了表征. 采用 X 射线单晶衍射技术测定了 Schiff 碱大环 3 的晶体 结构, 结果表明大环 3 具有扭曲的 “8” 字形结构. 采用 UV-vis 光谱滴定技术对大环与系列阴离子的键合作用进行了 考察, 结果表明, Schiff 碱大环 3 对 $\mathrm{F}^{-}$离子有明显的选择性识别作用, 并测定了该配位反应的配位比和平衡常数. 关键词 大环化合物; 合成; 晶体结构; 识别
\end{abstract}

\section{Synthesis, Structures of Macrocyclic Compounds Containing Imide Skeleton and the Study on Recognition for $\mathrm{F}^{-}$Ion}

\author{
Wang, Fangfang ${ }^{a} \quad \mathrm{Ou}, \mathrm{Min}^{a} \quad{\text { Deng, } \text { Yaxin }^{a}} \quad \mathrm{Ran}, \mathrm{Xu}^{a} \quad$ Zhang, Qilong $^{b} \quad$ Zhu, Bixue ${ }^{a, *}$ \\ ( ${ }^{a}$ Key Laboratory of Macrocyclic and Supramolecular Chemistry, Guizhou University, Guiyang 550025) \\ ( ${ }^{b}$ Department of Chemistry, Guiyang Medical College, Guiyang 550004)
}

\begin{abstract}
A novel [1+1] Schiff base macrocyclic compound 3 has been synthesized from precursors $N, N^{\prime}$-(2-aminophenyl)pyridine-2,6-dicarboxamide (1) and 1,2-bis(2'-formacylphenoxy)tetrane (2) via condensation in the presence of the phosphoric acid. Furthermore the macrocycle 3 was reduced to the corresponding saturated macrocycle 4 . Both macrocycles were characterized by ${ }^{1} \mathrm{H}$ NMR, IR, MS and elemental analysis. The crystal structure of $\mathbf{3}$ was determined by X-ray diffraction analysis. The result reveals that the macrocycle $\mathbf{3}$ exhibits twisted to "figure eight" conformation. The results show that the macrocycle 3 displays a selective recognition ability for $\mathrm{F}^{-}$ion by the both macrocycles with a series of anions using UV-vis absorption spectra technique. The stoichiometric ratio and the stability constant of the coordination reaction were determined.

Keywords macrocyclic compound; synthesis; crystal structure; recognition
\end{abstract}

新型阴离子受体的合成与设计一直是备受关注的 研究领域 ${ }^{[1,2]}$. 研究表明, 含 2,6-酰亚胺吡啶骨架的有机 配体，因其中氮原子易于同阴离子之间形成氢键作用， 是一类重要的阴离子受体 ${ }^{[3,4]}$. 特别是设计合成含 2,6-酰 亚胺吡啶骨架的有机大环配体, 通过环腔尺寸或大环结 构刚、柔性改变, 实现其对不同阴离子的选择性识别作 用, 一直是富有挑战性的研究课题 ${ }^{[5,6]}$.

据此, 本文先合成前体二胺 $N, N^{\prime}$-(2-胺基苯基)-2,6二甲酰亚胺吡啶(1)和前体二醛 1,4-二(2'-甲酰苯氧基)丁 烷(2), 再将两前体缩合得到[1+1] Schiff 碱大环化合物
3 , 进一步采用 $\mathrm{NaBH}_{4}$ 将 Schiff 碱大环 3 还原得到结构 更具柔性的饱和大环 4 . 同时, 采用 $\mathrm{X}$ 单晶衍射技术对 大环化合物 3 的晶体进行了结构解析，并分别考察了大 环 3 和 4 与系列阴离子的配位作用性质(大环化合物合 成路线见 Scheme 1).

\section{1 实验部分}

\section{1 仪器和试剂}

Bio-Rad 型傅立叶红外光谱仪 $\left(4000 \sim 400 \mathrm{~cm}^{-1}\right)$; Ry-2 型熔点仪(温度计未校正); JEOL ECX $400 \mathrm{MHz}$ 核

\footnotetext{
*E-mail: sci.bxzhu@gzu.edu.cn

Received July 21, 2013; revised September 27, 2013; published online October 31, 2013.

Project supported by the National Natural Science Foundation of China (No. 21061003) and the International Cooperation Foundation of Guizhou Province [No. (2009)700104].

国家自然科学基金(No. 21061003)和贵州省国际合作基金[No. (2009)700104]资助项目.
} 


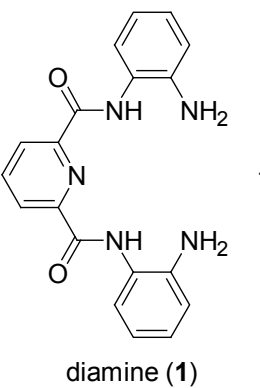

diamine (1)

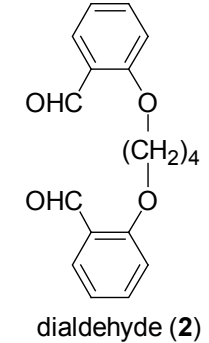

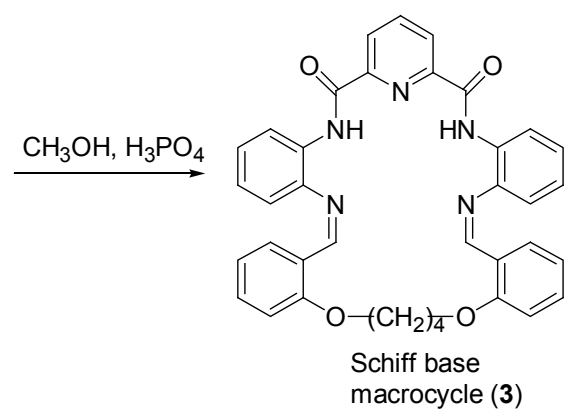

macrocycle (3)

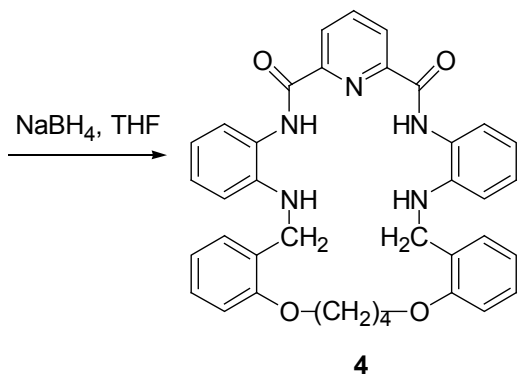

Scheme 1

磁共振仪; Vario EL III 型元素分析仪(德国); 岛津 UV-1800 紫外分光光度计(日本); MS (Angilent 1100)质 谱仪; 晶体结构测定采用 Bruker Smart Apex 衍射仪.

2,6-吡啶二羧酸、1,4-二溴丁烷(阿拉丁化学试剂有 限公司), 水合肼 $(80 \%$, 天津市科密欧化学试剂有限公 司), 氯化亚砜、邻硝基苯胺、水杨醛及其它试剂均为市 售分析纯.

\section{2 合成方法}

\subsubsection{Schiff 碱大环 3 的合成}

前体二胺 $N, N^{\prime}$-(2-胺基苯基)-2,6-二甲酰亚胺吡啶(1) 的合成: 按照文献[7]方法合成前体二胺 $N, N^{\prime}$-(2-胺基苯 基)-2,6-二甲酰亚胺吡啶(1), 黄色固体, 经真空干燥、称 量, 产率 $76 \%$. m.p. $203 \sim 205{ }^{\circ} \mathrm{C} ;{ }^{1} \mathrm{H}$ NMR (DMSO- $d_{6}$, $400 \mathrm{MHz}) \delta: 10.68$ (s, 2H, NH), 8.29 8.31 (m, 3H, PyH), $6.60 \sim 7.15(\mathrm{~m}, 8 \mathrm{H}, \mathrm{ArH}), 5.10\left(\mathrm{~s}, 4 \mathrm{H}, \mathrm{NH}_{2}\right)$.

前体二醛 1,4-二(2'-甲酰苯氧基)丁烷(2)的合成: 分 别称取水杨醛 $12.2 \mathrm{~g}(0.1 \mathrm{~mol})$ 和 1,4 -二溴丁烷 $10.8 \mathrm{~g}$ $(0.05 \mathrm{~mol})$ 溶解于 $100 \mathrm{~mL} \mathrm{DMF}$ 中, 转入 $250 \mathrm{~mL}$ 的三口 烧瓶, $\mathrm{N}_{2}$ 保护, 恒温 $80{ }^{\circ} \mathrm{C}$, 搅拌 $12 \mathrm{~h}$. 将反应液倒入冰 水浴中, 静置、过滤, 固体粗产品用 $95 \%$ 的乙醇重结晶, 得到 1,4-二(2'-甲酰苯氧基)丁烷的白色晶体 $17.9 \mathrm{~g}$, 产率 为 $78 \%$ m.p. $114 \sim 115{ }^{\circ} \mathrm{C} ;{ }^{1} \mathrm{H}$ NMR (DMSO- $d_{6}, 400$ MHz) $\delta: 10.45$ (s, 2H, CHO), 7.82 7.84 (m, 2H, ArH), $7.53 \sim 7.57(\mathrm{~m}, 2 \mathrm{H}, \mathrm{ArH}), 7.04(\mathrm{t}, J=7.2 \mathrm{~Hz}, 2 \mathrm{H}, \operatorname{ArH})$, $7.00(\mathrm{~d}, J=8.4 \mathrm{~Hz}, 2 \mathrm{H}, \mathrm{ArH}), 4.21(\mathrm{~d}, J=4.8 \mathrm{~Hz}, 4 \mathrm{H}$, $\left.\mathrm{OCH}_{2}\right), 2.11\left(\mathrm{t}, J=2.8 \mathrm{~Hz}, 4 \mathrm{H}, \mathrm{CH}_{2}\right)$.

Schiff 碱大环 3 的合成: 在 $250 \mathrm{~mL}$ 的三口瓶中, 向 $100 \mathrm{~mL}$ 的甲醇中加入 $N, N^{\prime}$-(2-胺基苯基)-2,6-二甲酰亚 胺吡啶 $(0.348 \mathrm{~g}, 1 \mathrm{mmol})$, 搅拌溶解. 向溶液中滴加 2 滴 磷酸, 搅拌、混匀后, 缓慢滴加 $0.298 \mathrm{~g}(1 \mathrm{mmol})$ 的二醛 1,4-二(2'-甲酰苯氧基)丁烷(2)的甲醇溶液 $50 \mathrm{~mL}$, 反应 混合液中有沉淀生成, 继续反应 $24 \mathrm{~h}$. 抽滤, 滤饼用甲 醇洗涤 3 5 次, 用丙酮重结晶, 经真空干燥得浅黄色固 体 30.413 g, 产率 64\%. m.p. $313 \sim 315{ }^{\circ} \mathrm{C} ;{ }^{1} \mathrm{H}$ NMR $\left(\mathrm{CDCl}_{3}, 400 \mathrm{MHz}\right) \delta: 11.01$ (s, 2H, $\left.\mathrm{CONH}\right), 8.69$ (d, $J=$
$7.6 \mathrm{~Hz}, 2 \mathrm{H}, \mathrm{CH}=\mathrm{N}), 8.53(\mathrm{~d}, J=8.0 \mathrm{~Hz}, 2 \mathrm{H}, \mathrm{PyH}), 8.30$ (s, 2H, ArH), 8.17 (t, $J=7.6 \mathrm{~Hz}, 1 \mathrm{H}, \mathrm{PyH}), 7.98 \sim 7.96(\mathrm{~m}$, $2 \mathrm{H}, \mathrm{ArH}), 7.29(\mathrm{~s}, 1 \mathrm{H}, \mathrm{ArH}), 6.99 \sim 6.95(\mathrm{~m}, 2 \mathrm{H}, \mathrm{ArH})$, $6.84(\mathrm{~d}, J=7.2 \mathrm{~Hz}, 2 \mathrm{H}, \mathrm{ArH}), 6.70 \sim 6.65(\mathrm{~m}, 5 \mathrm{H}, \mathrm{ArH})$, $6.33 \sim 6.26$ (m, $2 \mathrm{H}, \mathrm{ArH}), 4.30$ (s, $4 \mathrm{H}, \mathrm{OCH}_{2}$ ), $2.20 \sim 2.09$ (m, 4H, $\left.\mathrm{CH}_{2}\right)$; IR ( $\left.\mathrm{KBr}\right) v: 3307(\mathrm{CON}-\mathrm{H}), 1680(\mathrm{C}=\mathrm{O})$, 1587, 1524, $1453(\mathrm{C}=\mathrm{C}), 1249(\mathrm{C}-\mathrm{O}), 1618(\mathrm{C}=\mathrm{N})$ $\mathrm{cm}^{-1}$; FABMS $m / z$ : $634[\mathrm{M}+\mathrm{Na}]^{+}$. Anal. calcd for $\mathrm{C}_{37} \mathrm{H}_{31} \mathrm{~N}_{5} \mathrm{O}_{4}$ : C 72.89, H 5.13, N 11.49; found C 72.67, H 5.36, N 11.31 .

取少量固体 3 溶解于乙酸乙酯和二氯甲烷 $(V: V=$ $1: 1)$ 的混合溶剂中, $3 \mathrm{~d}$ 后长出适合 $\mathrm{X}$ 射线单晶衍射测 定的黄色块状晶体.

\subsection{2 大环 4 的合成}

称取 Schiff 碱大环 $30.5 \mathrm{~g}(0.82 \mathrm{mmol})$ 溶解于 $50 \mathrm{~mL}$ 四氢呋喃中, 在不断摚拌下, 滴加 2 滴冰醋酸, 分三次 加入 $\mathrm{NaBH}_{4}(0.15 \mathrm{~g}, 4.0 \mathrm{mmol})$, 常温反应 $8 \mathrm{~h}$. 旋去大部 分溶剂, 加入 $50 \mathrm{~mL}$ 蒸馏水, 用氯仿萃取得黄色固体大 环 $40.22 \mathrm{~g}$, 产率 44\%. m.p. 243 245 ${ }^{\circ} \mathrm{C}$; ${ }^{1} \mathrm{H}$ NMR $\left(\mathrm{CDCl}_{3}, 400 \mathrm{MHz}\right) \delta: 9.82$ (s, 2H, CONH), 8.52 (d, $J=7.6$ $\mathrm{Hz}, 2 \mathrm{H}, \mathrm{PyH}), 8.14$ (t, $J=7.6 \mathrm{~Hz}, 1 \mathrm{H}, \mathrm{PyH}), 7.95$ (d, $J=$ $7.2 \mathrm{~Hz}, 2 \mathrm{H}, \mathrm{ArH}), 7.10 \sim 7.04$ (m, 6H, ArH), 7.01 (d, $J=$ $7.6 \mathrm{~Hz}, 1 \mathrm{H}, \mathrm{ArH}), 6.95$ (t, $J=7.6 \mathrm{~Hz}, 2 \mathrm{H}, \mathrm{ArNH}), 6.81$ (d, $J=7.2 \mathrm{~Hz}, 2 \mathrm{H}, \mathrm{ArH}), 6.73 \sim 6.68(\mathrm{~m}, 5 \mathrm{H}, \mathrm{ArH}), 4.21$ (s, $\left.4 \mathrm{H}, \mathrm{ArCH}_{2}\right), 4.07$ (d, J=4.4 Hz, 4H, $\left.\mathrm{OCH}_{2}\right), 1.98(\mathrm{~s}, 4 \mathrm{H}$, $\left.\mathrm{CH}_{2}\right)$; IR $(\mathrm{KBr}) v: 3345(\mathrm{CON}-\mathrm{H}), 1677(\mathrm{C}=\mathrm{O}), 1599$, 1539, $1487(\mathrm{C}=\mathrm{C}), 1240(\mathrm{C}-\mathrm{O}), 1161(\mathrm{C}-\mathrm{N}) \mathrm{cm}^{-1}$; FABMS $m / z$ : $636[\mathrm{M}+\mathrm{Na}]^{+}$. Anal. calcd for $\mathrm{C}_{37} \mathrm{H}_{35} \mathrm{~N}_{5} \mathrm{O}_{4}$ : C 72.41, H 5.75, N 11.41; found C 72.57, H 5.94, N 11.31.

\section{3 晶体结构测定}

选取大小适当的晶体, 用 Bruker Smart Apex 单晶衍 射仪, 采用经石墨单色器单色化的 Mo K $\alpha$ 射线 $(\lambda=$ $0.071073 \mathrm{~nm})$, 以 $\varphi-\omega$ 扫描方式收集单晶衍射数据. 强 度数据进行了经验吸收校正、LP 校正. 晶体结构由直接 法解得. 对全部非氢原子坐标及其各向异性热参数进行 
了全矩阵最小二乘法修正. 所有计算用 SHELX-97 程序 完成 ${ }^{[8]}$.

\section{4 离子识别试验}

\subsection{1 大环与系列阴离子作用体系的 UV-vis 光谱}

分别移取 $1 \mathrm{~mL}$ 浓度为 $1 \times 10^{-4} \mathrm{~mol} \cdot \mathrm{L}^{-1}$ 的 Schiff 碱 大 3 和饱和大环 4 的 $\mathrm{CH}_{3} \mathrm{CN}$ 溶液于一系列 $10 \mathrm{~mL}$ 容量 瓶中, 分别加入等量、浓度均为 $1 \times 10^{-3} \mathrm{~mol} \cdot \mathrm{L}^{-1}$ 的系列 阴离子(即 $\mathrm{F}^{-}, \mathrm{Cl}^{-}, \mathrm{Br}^{-}, \mathrm{I}^{-}, \mathrm{NO}_{3}{ }^{-}, \mathrm{HSO}_{4}{ }^{-}, \mathrm{H}_{2} \mathrm{PO}_{4}{ }^{-}, \mathrm{ClO}_{4}{ }^{-}$, $\left.\mathrm{OAc}^{-}\right)$的 TBA 盐 $\left(\mathrm{TBA}^{+} \mathrm{X}^{-}\right)$的乙腈溶液, 用 $\mathrm{CH}_{3} \mathrm{CN}$ 稀释 至刻度, 混匀, 放置达体系吸光度稳定不变后, 室温下 在 200 600 nm 范围内记录各反应体系的紫外-可见吸 收光谱 $\left(\mathrm{CH}_{3} \mathrm{CN}\right.$ 作参比).

1.4.2 Schiff 碱大环 3 与 $\mathrm{F}^{-}$离子配位反应的配位比及 稳定常数

Schiff 碱大环 3 与 $\mathrm{F}^{-}$离子的 UV-vis 光谱滴定: 室温 下, 分别移取 $1 \mathrm{~mL}$ 浓度为 $1.00 \times 10^{-4} \mathrm{~mol} \cdot \mathrm{L}^{-1}$ 的大环 3 的乙腈溶液, 加入系列 $10 \mathrm{~mL}$ 的容量瓶中, 向其中分别 滴加浓度为 $1.00 \times 10^{-3} \mathrm{~mol} \cdot \mathrm{L}^{-1}$ 、不同体积的 $\mathrm{F}^{-}$阴离子 的 TBA 盐 $\left(\mathrm{TBA}^{+} \mathrm{X}^{-}\right)$的乙腈溶液, 以乙腈定容, 得到 $\mathrm{F}^{-}$ 浓度依次增加的系列反应体系 $\left(\left[\mathrm{F}^{-}\right] /[3]\right.$ 的物质的量比为 0 5 倍). 待反应达平衡后, 在 $200 \sim 600 \mathrm{~nm}$ 范围内进行 UV-vis 光谱扫描, 通过等摩尔连续变化法确定配位反应 的配位比.

进一步采用 Job 法对主客体配位比进行确定, 即在 乙腈溶液中, 分别配置浓度均为 $5.00 \times 10^{-4} \mathrm{~mol} \cdot \mathrm{L}^{-1}$ 大 环 3 和 $\mathrm{F}^{-}$离子的乙腈溶液作为操作液. 固定各配位反应 体系中反应物总浓度为 $5.00 \times 10^{-5} \mathrm{~mol} \cdot \mathrm{L}^{-1}$ 的条件下， 配置系列大环 3 和 $\mathrm{F}^{-}$离子浓度比不同的反应体系. 待系 列体系达反应平衡后，测定系列体系的吸光度.

\section{2 结果与讨论}

\section{1 大环化合物合成}

合成席夫碱大环的方法较多, 文献报道方法主要分 为四种, 即高度稀释合成法 ${ }^{[9]}$ 、金属离子模板合成 法 $^{[10,11]}$ 、阴离子模板合成法 ${ }^{[12]}$ 以及酸催化合成法 ${ }^{[13]}$ 等. 本文在目标大环 3 的合成过程中, 分别采用高度稀释法 和酸催化合成法对大环 3 的合成进行尝试. 结果表明, 在稀溶液中, 即使是无酸催化作用条件下, 也可得到目 标大环 3, 但产率较低(34.1\%). 在酸催化合成过程中, 分别考察了不同的酸(如盐酸、硫酸、硝酸、磷酸、乙酸 等)对反应的催化效果, 结果如表 1. 数据表明, 在反应 体系中分别加入硫酸或磷酸时, 目标大环的产率明显提 高, 并以磷酸的催化作用最为有效, 不仅增加产率, 也 加快反应速率.
表 1 不同酸对产率的影响

Table 1 The yields of macrocycles in the presence of the different acids

\begin{tabular}{ccccccc}
\hline 酸 & 不加酸 & 盐酸 & 硝酸 & 乙酸 & 硫酸 & 磷酸 \\
\hline 产率 $/ \%$ & 34.1 & 30.7 & 28.9 & 34.2 & 51.2 & 64.3 \\
\hline
\end{tabular}

\section{2 谱学表征}

\subsection{1 ${ }^{1} \mathrm{H}$ NMR 和质谱}

前体二醛 1 和二胺 2 进行缩合作用得到 Schiff 碱大 环化合物 3 后，在 Schiff碱大环 3 中，原料二醛在 $\delta 10.45$ 处的醛基质子峰消失，二胺化合物在 $\delta 10.68$ 处的胺基 质子峰消失, 大环 3 在 $\delta 8.69$ 处出现两个 $\mathrm{N}=\mathrm{CH}$ 的质子 峰，表明前体 1 中两个胺基均与化合物 2 中两个醛基 发生了全缩合作用. 在 $\delta 11.01$ 处出现两个酰亚胺 $(\mathrm{CONH})$ 的质子峰, 同时在 $\delta 4.30$ 处呈现出烷氧链 $\left(\mathrm{OCH}_{2}\right)$ 的 4 个亚甲基质子峰，在 $\delta 2.09 \sim 2.20$ 区域呈现 出烷基链中 4 个亚甲基质子 $\left(\mathrm{CH}_{2}\right)$ 峰. 对大环 3 进行质 谱(FABMS)表征，其分子离子峰 $[\mathrm{M}+\mathrm{Na}]^{+} \mathrm{m} / \mathrm{z}$ 为 $632.3,633.3,634.3$, 数据表明前体二胺 1 和二醛 $\mathbf{2}$ 按 1 : 1 的物质的量比缩合形成了 $[1+1]$ 的 Schiff 碱大环化合 物 $\mathbf{3}$, 结果与 $\mathbf{3}$ 的晶体结构相吻合.

进一步将 Schiff 碱大环 3 中的 $\mathrm{N}=\mathrm{C}$ 双键经 $\mathrm{NaBH}_{4}$ 还原后得饱和大环 4 . 在 4 的 ${ }^{1} \mathrm{H}$ NMR 中, 两个酰亚胺 $(\mathrm{CONH})$ 的质子峰出现在 $\delta 9.82$ 处, 与大环 3 中相应质子 的化学位移 $(\delta$ 11.01)相比向高场发生了位移, 这可能源 于在大环 3 中酰亚胺 $(\mathrm{CONH})$ 质子与相邻 Schiff 碱 $\mathrm{N}=\mathrm{C}$ 中氮原子之间形成的分子内氢键. 相应的还原大环 4 中, 在 $\delta 6.95$ 处出现了由 Schiff 碱 $\mathrm{N}=\mathrm{C}$ 双键被还原产生的 亚胺质子峰 $(\mathrm{C}-\mathrm{NH})$, 相应的 2 个次甲基质子峰 $\left(\mathrm{CH}_{2}-\right.$ $\mathrm{N})$ 出现在 $\delta 4.21$ 处. 同时, 在 $\delta 4.07$ 处呈现烷氧链 $\left(\mathrm{OCH}_{2}\right)$ 的 4 个亚甲基质子峰, 在 $\delta 1.98$ 处呈现出烷基链 上的 4 个亚甲基 $\left(\mathrm{CH}_{2}\right)$ 质子峰. 对大环 4 进行质谱 (FABMS)表征，其分子离子峰 $[\mathrm{M}+\mathrm{Na}]^{+} \mathrm{m} / \mathrm{z}$ 为 636.3 , 637.3，638.3. ${ }^{1} \mathrm{H}$ NMR 和质谱表征结果均与饱和大环 4 的结构吻合.

\subsubsection{IR 光谱和 UV-vis 光谱}

Schiff 碱大环 3 的红外光谱数据表明，原料二醛在 $1686 \mathrm{~cm}^{-1}$ 的醛基 $(\mathrm{HC}=\mathrm{O})$ 振动峰消失. 在 $1618 \mathrm{~cm}^{-1}$ 处 出现了很强的 Schiff 碱 $\mathrm{C}=\mathrm{N}$ 双键的伸缩振动峰. 大环 3 还原为大环 4 后, 大环 3 中在 $1618 \mathrm{~cm}^{-1}$ 附近的 $\mathrm{C}=\mathrm{N}$ 双 键伸缩振动峰消失, 在 $1161 \mathrm{~cm}^{-1}$ 处呈现 $(\mathrm{C}-\mathrm{N})$ 振动 峰. 大环 3 和大环 4 的羰基 $(\mathrm{C}=\mathrm{O})$ 振动峰分别出现在 1678 和 $1677 \mathrm{~cm}^{-1}$ 处, 相应于酰亚胺基团中的亚胺( $\mathrm{N}-$ $\mathrm{H}$ )振动峰分别出现在 3334 和 $3345 \mathrm{~cm}^{-1}$ 处.

在乙腈溶剂中, Schiff 碱大环 3 的电子吸收光谱最 大吸收峰分别出现在 238,274 和 $358 \mathrm{~nm}$ 三处, 大环 4 
的最大吸收峰出现在 245 和 $275 \mathrm{~nm}$ 两处. 大环 3 在 238 和 $274 \mathrm{~nm}$ 两处的吸收, 大环 4 在 245 和 $275 \mathrm{~nm}$ 两处的 吸收均由分子中芳环的 $\pi-\pi *$ 跃迁所致. 对比大环 3 和大 环 4 的 UV-vis 光谱可见, 在大环 3 中相应于 $\mathrm{C}=\mathrm{N}$ 的 $\pi-\pi *$ 跃迁吸收在大环 4 的吸收光谱中已经消失, 表明大 环 4 中的 Schiff 碱 $\mathrm{C}=\mathrm{N}$ 双键已经被还原 ${ }^{[14]}$.

\subsection{Schiff 碱大环 3 的晶体结构描述}

通过单晶培养和结构解析, 获得了大环 $\mathbf{3}$ 的晶体结 构数据(见辅助材料中表 1 和表 2). 大环 3 的晶体结构数 据存于英国剑桥数据中心, CCDC 为 950321 .

[1+1] Schiff 碱大环 3 分子结构如图 1a. 在该大环 分子中, $\mathrm{C}(28)=\mathrm{N}(3)$ 和 $\mathrm{C}(21)=\mathrm{N}(4)$ 的键长分别为 $0.1275(2)$ 和 $0.1275(2) \mathrm{nm}$, 接近于典型的碳氮双键得到 键长(辅助材料表 2 , 典型的 $\mathrm{C}=\mathrm{N}$ 双键的平均键长为 $\left.0.126 \mathrm{~nm}^{[15]}\right)$. 大环分子的中心吡啶环氮原子 $\mathrm{N}(1)$ 分别 与其两臂的 Schiff 碱 $(\mathrm{C}=\mathrm{N})$ 氮原子通过位于其间的酰亚 胺质子形成桥连氢键(如图 $1 \mathrm{~b}$, 氢键参数见辅助材料表 $3)$, 导致大环分子呈现一扭曲的 “ 8 ” 字构型. 位于中央 吡啶环两臂包含酰亚胺骨架的间隔基 $\mathrm{C}(34)-\mathrm{N}(2)-$ $\mathrm{C}(35)-\mathrm{C}(36)$ 和 $\mathrm{C}(5)-\mathrm{N}(5)-\mathrm{C}(4)-\mathrm{C}(3)$ 的扭曲角分别 为 $172.46(2)^{\circ}$ 和 $169.54(2)^{\circ}$, 即处在吡啶环两臂的这 2 个 苯环 $\mathrm{Cg} 1[\mathrm{C}(5)-\mathrm{C}(10)]$ 和 $\mathrm{Cg} 2[\mathrm{C}(29)-\mathrm{C}(34)]$ 处于相互 扭曲的位置, 苯环 $\mathrm{Cg} 1$ 和苯环 $\mathrm{Cg} 2$ 之间的二面角为

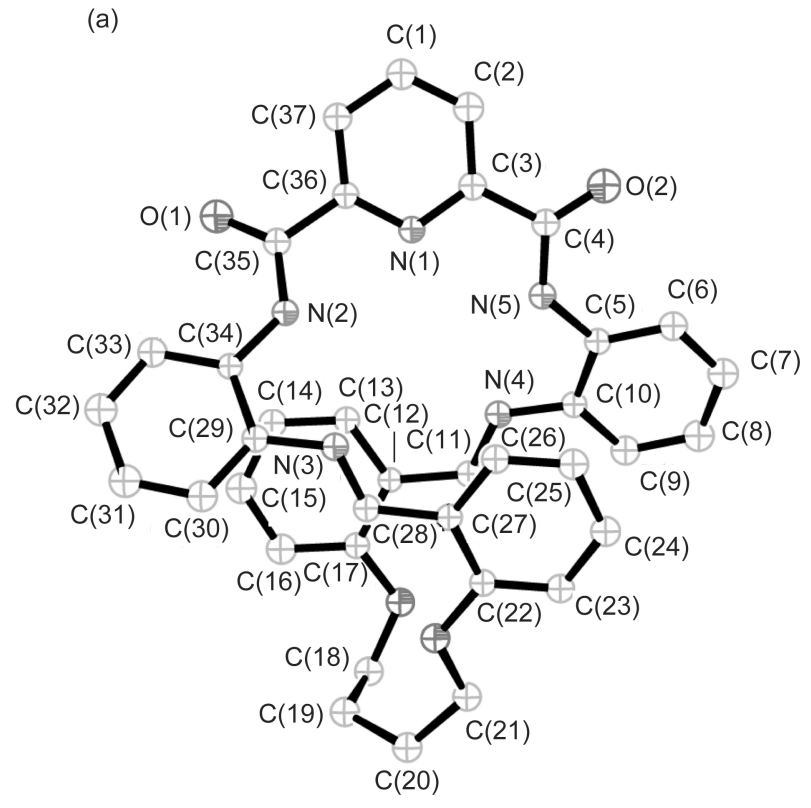

$158.6^{\circ}$. 经由烷氧链连接的两个苯环 $\mathrm{Cg} 3$ [C(12)- $\left.\mathrm{C}(17)\right]$ 和 $\mathrm{Cg} 4[\mathrm{C}(22)-\mathrm{C}(27)]$ 之间的二面角 $169.3^{\circ}$. Schiff 碱 $[\mathrm{HC}(28)=\mathrm{N}]$ 中碳上的质子与苯环 $\mathrm{Cg} 3[\mathrm{C}(12)-\mathrm{C}(17)]$ 之 间存在 $\mathrm{C}-\mathrm{H}(28) \cdots \pi$ 相互作用 $[\mathrm{H}(28) \cdots \mathrm{Cg} 3=0.3306 \mathrm{~nm}$, $\left.\mathrm{C}(28) \cdots \mathrm{Cg} 3=0.3552(4) \mathrm{nm}, \quad \mathrm{C}-\mathrm{H}(28) \cdots \mathrm{Cg} 3=97.67^{\circ}\right]$, 使得该扭曲的分子骨架得到进一步稳定.

\section{4 大环与阴离子配位作用考察}

\subsection{1 大环与系列阴离子作用体系的 UV-vis 光谱}

通过 Schiff 碱大环 3 和 4 分别与系列阴离子(即 $\mathrm{F}^{-}$, $\mathrm{Cl}^{-}, \mathrm{Br}^{-}, \mathrm{I}^{-}, \mathrm{NO}_{3}^{-}, \mathrm{HSO}_{4}^{-}, \mathrm{H}_{2} \mathrm{PO}_{4}^{-}, \mathrm{ClO}_{4}^{-}, \mathrm{OAc}^{-}$)配 位作用体系的 UV-vis 光谱扫描, 结果表明 Schiff 碱大环 3 仅对 $\mathrm{F}^{-}$具有较明显的配位作用(图 2a), 与其它阴离子 均未能观察到明显作用. 饱和大环 4 则对所选用的系列 阴离子之间均未能观察到明显作用. 据此, 进一步测定 了大环 3 与 $\mathrm{F}^{-}$离子选择性识别作用的配位比及配位稳 定常数.

2.4.2 Schiff 碱大环 3 与 $\mathrm{F}^{-}$离子配位反应的配位比及 稳定常数

Schiff 碱大环 $3\left(1.00 \times 10^{-5} \mathrm{~mol} \cdot \mathrm{L}^{-1}\right)$ 与不同浓度的 $\mathrm{F}^{-}$离子作用体系 UV-Vis 光谱扫描如图 $2 b$, 从图 $2 b$ 可见， 随着体系中 $\mathrm{F}^{-}$浓度增加, 体系的吸光度在 $233,269 \mathrm{~nm}$ 两处依次上升, 曲线在 $249,260,295,345 \mathrm{~nm}$ 处呈现 4 个等吸收点.

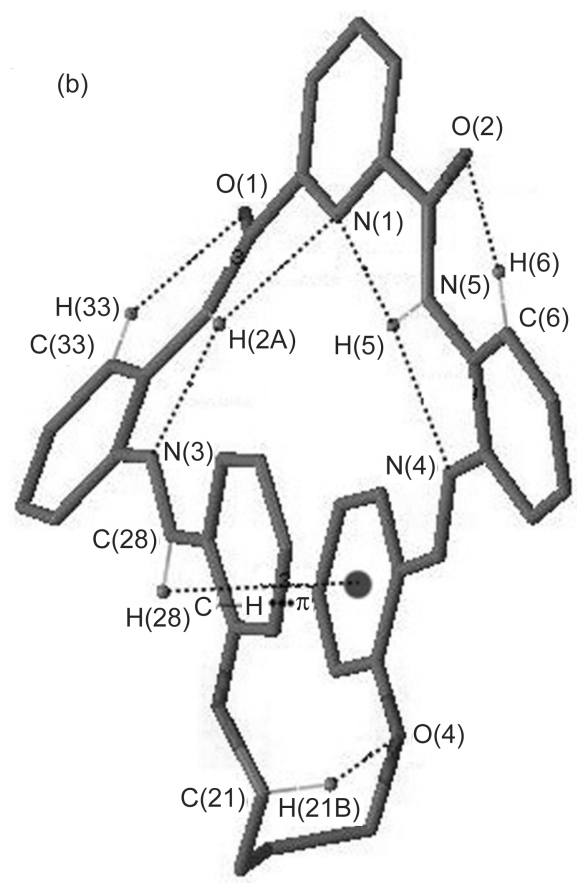

图 1 (a) Schiff 碱大环 3 的分子结构(栯球几率为 $20 \%$ )及(b)大环分子内氢键及 C- $-\mathrm{H} \cdots \pi$ 相互作用

Figure 1 (a) Molecular structure of the Schiff base macrocycle 3 (probability of ellipsoid is 20\%) and (b) interactions of the intramolecular hydrogen bonds and $\mathrm{C}-\mathrm{H} \cdots \pi$ interaction in $\mathbf{3}$ 

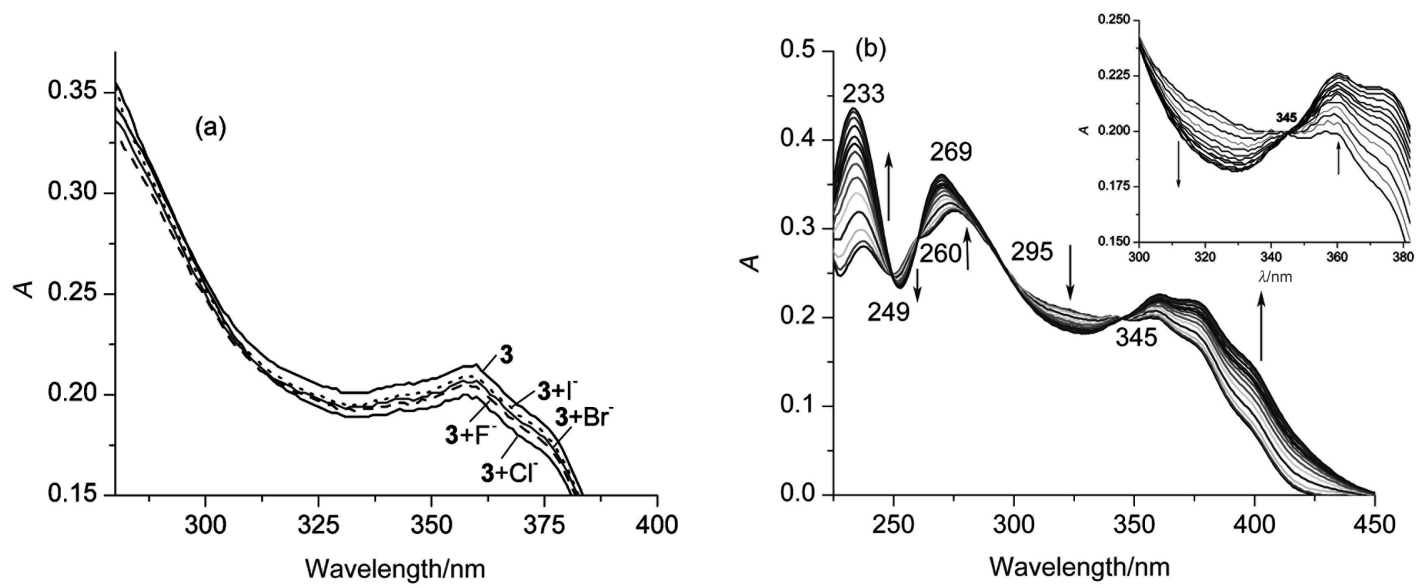

图 2 (a)大环 3 与卤阴离子 $\left(\mathrm{F}^{-}, \mathrm{Cl}^{-}, \mathrm{Br}^{-}, \mathrm{I}^{-}\right)$作用体系的紫外-可见吸收光谱及(b)大环 $\mathbf{3}$ 与 $\mathrm{F}^{-}$反应体系的 UV-vis 吸收光谱滴定曲 线

Figure 2 (a) UV-vis absorption spectra of the macrocycle 3 in the presence of various $\mathrm{X}^{-}$ions $\left(\mathrm{F}^{-}, \mathrm{Cl}^{-}, \mathrm{Br}^{-}, \mathrm{I}^{-}\right)$and (b) the titration curve of UV-vis absorption spectrum for the system involving macrocycle $\mathbf{3}$ with $\mathrm{F}^{-}$

配位比确定：首先采用摩尔比法测定了大环 3 与 $\mathrm{F}^{-}$ 作用的配位比. 实验中用选择性较高的 $358 \mathrm{~nm}$ 波长处, 各作用体系吸光度 $A$ 对大环与 $\mathrm{F}^{-}$的摩尔比 $\left(N_{3} / \mathrm{N}_{\mathrm{F}}^{-}\right)$作图 (如图 3a). 可见, 当大环 $\mathbf{3}$ 与 $\mathrm{F}^{-}$配位键合的物质的量比
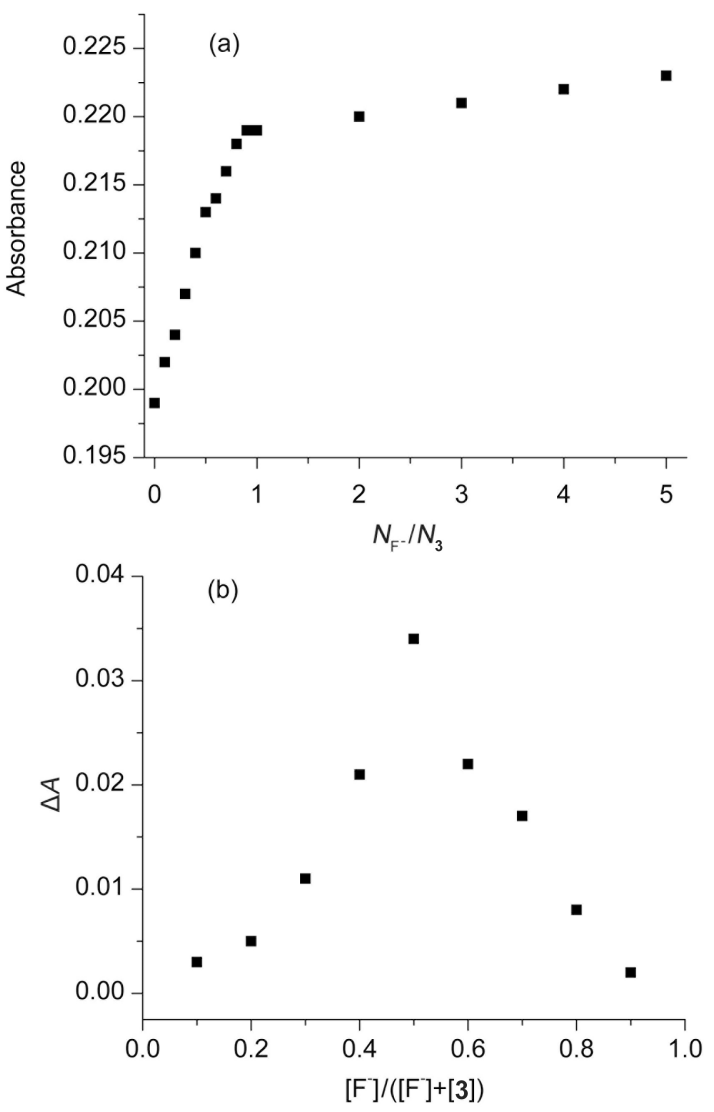

图 3 (a)大环 3 与 $\mathrm{F}^{-}$配位反应体系的 $A$ 与 $\left[N_{\mathrm{F}}^{-}\right] /\left[N_{3}\right]$ 的关系及 (b) Job 图

Figure 3 (a) Plot of $A$ vs. $\left[N_{\mathrm{F}}^{-}\right] /\left[N_{3}\right]$ for the system involving macrocycle 3 with $\mathrm{F}^{-}$and Job plot (b)
为 $1: 1$ 时，体系吸光度值趋向平缓并出现转折，表明 $\mathbf{3}$ 与 $\mathrm{F}^{-}$形成了配位比为 $1: 1$ 的配合物。

进一步采用了 Job 法确定配位作用的配位比，将各

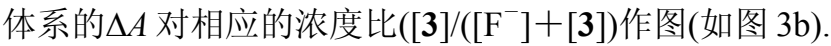
图 $3 \mathrm{~b}$ 表明大环 3 与 $\mathrm{F}^{-}$形成了 $1: 1$ 的配合物, 以上两种 方法测定所得配位比相互吻合.

稳定常数 $(K)$ 测定：按照文献[16]所报道的曲线拟合 方法，确定大环化合物 $\mathbf{3}$ 与 $\mathrm{F}^{-}$配位反应平衡常数. 实验 中, 选择性较高的 $358 \mathrm{~nm}$ 波长处各平衡体系的 $\Delta A$ 值和 相应的 $\mathrm{F}^{-}$浓度进行 $K$ 的计算，曲线拟合结果如图 4. 表

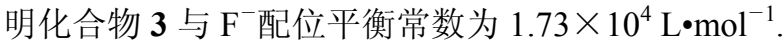

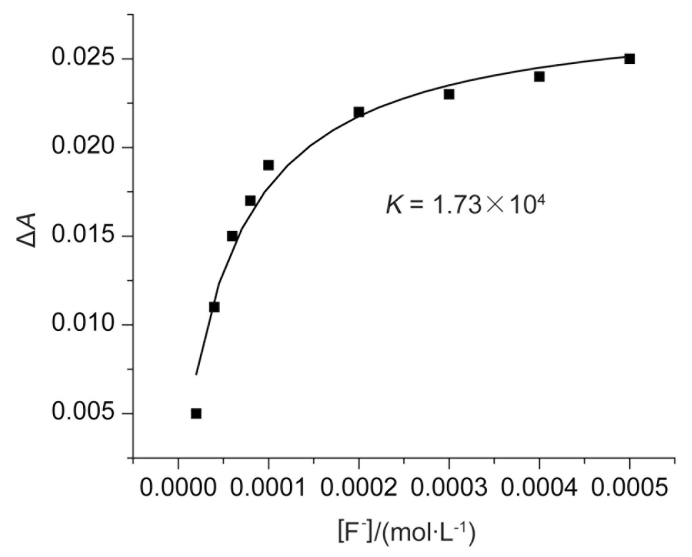

图 4 大环 3 和 $\mathrm{F}^{-}$相互作用体系的平衡常数的曲线拟合 $\left(\lambda_{\max }=358 \mathrm{~nm}\right)$

Figure 4 Curve fit of the equilibrium constant for the system involving macrocycle 3 with $\mathrm{F}^{-}$

\section{$2.5{ }^{1} \mathrm{H}$ NMR 滴定}

为进一步了解大环 3 与 $\mathrm{F}^{-}$离子键合作用的相关信 息，采用 ${ }^{1} \mathrm{H}$ NMR 滴定技术对体系中主客体作用性质进 
行考察, 结果如图 5.

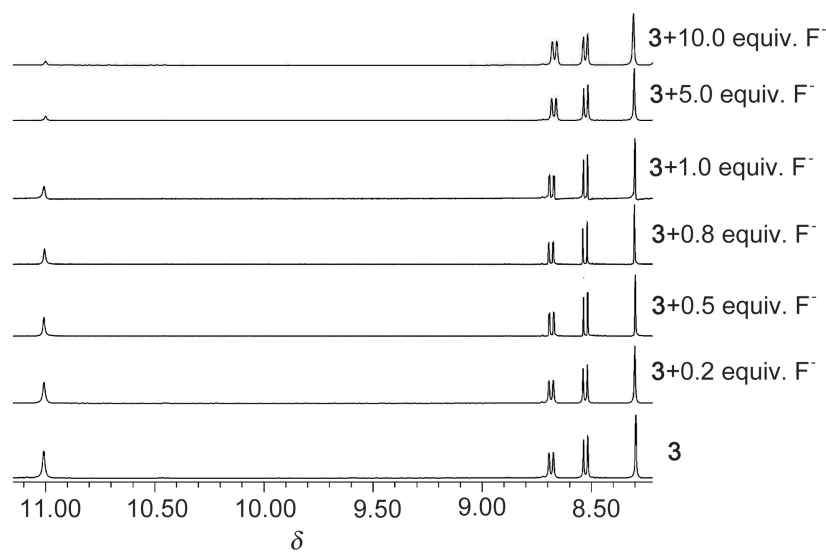

图 5 大环 3 对四丁基氟化铵 $(0 \sim 10$ equiv.)在氯仿溶液中的核 磁滴定局部图

Figure 5 Partial ${ }^{1} \mathrm{H}$ NMR titration of macrocycle 3 with $\mathrm{Bu}_{4} \mathrm{NF}$ $(0 \sim 10$ equiv. $)$ in $\mathrm{CDCl}_{3}$

从图中可以看出, 在主体大环 $\mathbf{3}$ 浓度不变的条件下, 随着 $\mathrm{F}^{-}$浓度的逐渐增加, 大环 3 的 $\mathrm{CH}=\mathrm{N}$ 质子峰 $(\delta$ 8.69)、 $\mathrm{PyH}$ 质子峰 $(\delta$ 8.53) 和 $\mathrm{ArH}$ 质子峰 $(\delta$ 8.30)的强度 和化学位移几乎未发生变化. 同样, 酰亚胺 $\mathrm{CONH}$ 质子 的 $\delta$ 也未能观察到明显的位移变化, 但其强度随 $\mathrm{F}^{-}$浓度 的增大明显减弱. 基于自由大环 3 中含有分子内氢键, 我们推测客体 $\mathrm{F}^{-}$应与主体大环 3 的酰亚胺基形成了氢 键 $\left(\mathrm{N}-\mathrm{H} \cdots \mathrm{F}^{-}\right)$作用.

\section{3 结论}

本文研究结果表明, 磷酸对催化合成目标大环 3 最 为有效. Schiff 碱大环 3 及其相应的还原产物 4 对所选用 的系列阴离子识别作用考察. 结果表明, 含酰亚胺骨架 的一类目标大环与阴离子之间发生氢键作用时, 大环结 构的刚柔性、酰亚胺氮原子与阴离子键合作用的取向, 以及客体阴离子大小、几何形状等因素, 对主客体之间 选择性识别能力存在较大影响.
辅助材料(Supporting Information) Schiff 碱大环 3 的 晶体结构数据以及 Schiff 碱大环 3 和 $\mathbf{4}$ 的 ${ }^{1} \mathrm{H}$ NMR, MS 和 UV-vis 图谱. 这些材料可以免费从本刊网站(http:// sioc-journal.cn/)上下载.

\section{References}

[1] Gale, P. A.; García-Garrido, S. E.; Garric, J. Chem. Soc. Rev. 2008, $37,151$.

[2] Kubik, S. Chem. Soc. Rev. 2009, 38, 585.

[3] Dorazco-González, A.; Höpfl, H.; Medrano, F.; Yatsimirsky, A. K. J. Org. Chem. 2010, 75, 2259.

[4] Sessler, J. L.; Katayev, E.; Pantos, G. D.; Ustynyuk, Y. A. Chem. Commun. 2004, 1276.

[5] Li, F.; Delgado, R.; Costa, J.; Drew, M. G. B.; Félix, V. Dalton Trans. 2005, 82.

[6] Hossain, M. A.; Kang, S. O.; Powell, D.; Bowman-James, K. Inorg. Chem. 2003, 42, 1397.

[7] Hu, P.; Guo, S.-Y.; Zhang, Q.-L.; Zhang, Y.-Q.; Zhu, B.-X. Chin. J. Org. Chem. 2013, 33, 325 (in Chinese).

(胡鹏, 郭思颖, 张奇龙, 张云黔, 朱必学, 有机化学, 2013, 33, 325.)

[8] Sheldrick, G. M. SHELX-97, University of Göttingen, Germany, 1997.

[9] Sellamuthu, A.; Kamalraj, S.; Varghese, B.; Johnpaul, M.; Muthusamy, K. Inorg. Chem. 2012, 51, 5580.

[10] Van Veggel, F. C. J. M.; Harkema, S.; Bas, M.; Verboom, W.; Van Staveren, C. J.; Gerritsma, G. J.; Reinhoudt, D. N. Inorg. Chem. 1989, 28, 1133.

[11] Van Staveren, C. J.; Van Eerden, J.; Van Veggel, F. C. J. M.; Harkema, S.; Reinhoudt, D. N. J. Am. Chem. Soc. 1988, 110, 4994.

[12] Ravikumar, I.; Pradyut, G. Chem. Soc. Rev. 2012, 41, 3077.

[13] Elwahy, A. H. M.; Masaret, G. S. J. Heterocycl. Chem. 2007, 44, 147.

[14] Zhu, B.-X.; Ruan, W.-J.; Yuan, R.-J.; Cao, X.-H.; Zhu, Z.-A. Chin. J. Appl. Chem. 2004, 10, 21 (in Chinese).

(朱必学, 阮文娟, 袁瑞娟, 曹小辉, 朱志昂, 应用化学, 2004, 10, 21.)

[15] Özkar, S.; Üıkü, D.; Yıldırım, L. T.; Biricik, N.; Gümgüm, B. J. Mol. Struct. 2004, 688, 207.

[16] Ou, M.; Deng, Y.-X.; Wang, F.-F.; Zhu, C.; Zhang, Q.-L.; Zhu, B. X. Chin. J. Org. Chem. 2013, 33, 1798 (in Chinese).

(欧敏, 邓雅欣, 王芳芳, 朱纯, 张奇龙, 朱必学, 有机化学, 2013, 33, 1798.) 